\title{
Prenatal Ultrasound Diagnosis of Double Aortic Arch versus \\ Right Aortic Arch Variant in Vascular Ring Formation - Case Report and Review of the Literature
}

Roxana Elena BOHILTEA ${ }^{a, b}$, Paul Costin PARIZAa ${ }^{a}$ Irina STAVARACHEc, Octavian MUNTEANUd, Vlad DIMA ${ }^{b}$, Bianca Margareta MIHAI ${ }^{b}$, Tiberiu Augustin GEORGESCUe, Eliza CINTEZA ${ }^{f}$

aDepartment of Obstetrics and Gynecology,

"Carol Davila" University of Medicine and Pharmacy Bucharest, Romania

bDepartment of Obstetrics, Gynecology and Neonatology, Filantropia Clinical Hospital, Bucharest, Romania

'Department of Radiology, Fundeni Clinical Institute, Bucharest, Romania

'Department of Anatomy, "Carol Davila" University of Medicine and Pharmacy,

Bucharest, Romania

eDepatment of Pathology, "Carol Davila" University of Medicine and Pharmacy,

Bucharest, Romania

fDepartment of Pediatric Cardiology,

"Carol Davila" University of Medicine and Pharmacy Bucharest,

"MS Curie" Emergency Clinical Hospital, Bucharest, Romania

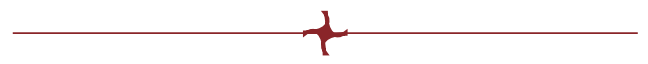

ABSTRACT
Double aortic arch represents a congenital vascular malformation that is characterized by the development
of a complete vascular ring around the esophagus and trachea due to an anomaly in the development
of branchial arteries. We present the case of a 31-year-old gravida that was referred for fetal ultrasound
anomalies screening at 22 weeks and six days of gestation. Routine ultrasound scanning of the fetus
revealed a structural aortic arch anomaly consistent with a double aortic arch, with no other cardiac and

Address for correspondence:

Paul Costin Pariza

Department of Obstetrics and Gynecology, "Carol Davila" University of Medicine and Pharmacy Bucharest, Dionisie Lupu 37, 020021, Bucharest, Romania Email:doinitapariza@gmail.com 
extracardiac congenital structural malformations. Knowledge of embryology and imaging spectrum of aortic arch anomalies that are able to form vascular rings around the trachea and esophagus are essential for an accurate antenatal diagnosis and therefore, for a correct clinical management.

Keywords: double aortic arch, vascular ring, prenatal diagnosis, ultrasound, right aortic arch.

\section{INTRODUCTION}

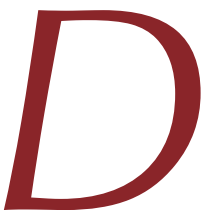

ouble aortic arch represents a congenital vascular malformation that is characterized by the development of a complete vascular ring around the esophagus and trachea due to an anomaly in the development of brachial arteries (1).

Although double aortic arch is a rare type of congenital malformation, affecting approximately $0.005-0.007 \%$ of fetuses, it is one of the most common congenital anomalies of the aortic arch system and, in $16.6 \%$ of cases, it can be associated with intracardiac malformations such as tetralogy of Fallot and transposition of the great arteries, atrial septal defect, ventricular septal defect and patent ductus arteriosus. In $24 \%$ of cases it can be associated with chromosomal abnormalities such as 22q11deletion $(2,3)$.

The vascular ring formed by the double aortic arch that encircles the trachea and esophagus can lead to important symptoms such as dyspnea, wheezing, stridor, cough, respiratory tract infections, and dysphagia (4). Rarely, crisis of apnea with respiratory arrest may occur.

Aortic embryology is a complex process and it begins during the third week of gestation, leading to six paired primitive aortic arches that develop between the ventral and dorsal aorta. Double aortic arch appears secondary to the persistence of both right and left arches and from each separate arch, carotid and subclavian arteries emerge; so, the left common carotid artery and left subclavian artery emerge from the left aortic arch, and the right common carotid artery and right subclavian artery emerge from the right arch (5). Most of cases (approximately $80 \%$ ) have a right dominant system, with a left aortic arch that is more frequently patent, but it can also be hypoplastic or atretic (4). Given that the descending aorta is usually opposite the dominant arch, a large right aortic arch, left-sided descending aorta and left sided ligamentum arteriosus is the most common presentation (5).
Prenatal imaging is required for an accurate antenatal diagnosis and it can be easily made by using ultrasonography. The diagnosis can be obtained in a transverse view of the fetal thorax at the level of the three-vessel and trachea view (6). The diagnosis of double aortic arch is made when there are two aortic arches that form a complete vascular ring encircling the trachea, with the common carotid artery and subclavian artery arising separately from each arch. After the diagnosis of double aortic arch is made it is important to determine whether there are any associated cardiac malformations (4).

The differential diagnoses of vascular ring causes have to include the following abnormal variants:

- right aortic arch with branching in mirror-image and a left ductus arteriosus arising from the retroesophageal dimple resulted from an atretic left arch of a double aortic arch anomaly;

- right aortic arch with aberrant left subclavian artery associating a left-sited ductus;

- left aortic arch associated with right descending aorta and also right ductus arteriosus;

- right aortic arch with left descending aorta and left ductus arteriosus;

- left aortic arch associating an aberrant right subclavian artery and also a right ductus arteriosus;

- right aortic arch with a retroesophageal left brachiocephalic trunk and a left ductus arteriosus.

Fetal MRI is also a useful tool for prenatal diagnosis, giving additional information about the cardiac function, intracardiac and vascular anatomy in a broad spectrum of congenital heart diseases (7). It can also give additional information about extracardiac anomalies (8).

Respiratory distress of the newborn is initially investigated by chest radiography, followed by computed tomography angiography or magnetic resonance angiography, which offer the three-di- 
mensional imaging of the vascular ring and provide anatomic details needed for corrective surgery. Echocardiography and bronchoscopy are complementary procedures. Surgical options include either minimally invasive techniques such as thoracoscopic or robotic-assisted surgery, or open procedures involving thoracotomy. Atretic or stenotic segment of one of the arches is the selected site for surgical division, the ductus or ligamentum section insuring the release of trachea from the decompressive ring.

The outcomes of surgical treatment are excellent, even though most patients have a complete resolution of symptoms after some time, complications occur in $11 \%$ of cases and $4 \%$ of infants die (9). Chronic respiratory symptoms are present and $42 \%$ of patients undergo surgery after three years of age (10). The reoperation rate is generally low, under $10 \%$.

\section{CASE REPORT}

41-year-old gravida 3 was referred for rouAtine fetal ultrasound at 22 weeks and six days of gestation. Routine ultrasound scanning of the male fetus revealed normal growth with a structural aortic arch anomaly consistent with double aortic arch, with no other cardiac and extracardiac congenital structural malformations. The right aortic arch was considered the principal one, the left arch being hypoplastic, similarly to the majority of cases from the literature (Figures 1-4). The amniotic fluid was normal and placenta was in normal position. Prenatal non-invasive testing was normal and so was the karyotype (Figure 5), which have been done due to the high rate of association of right aortic arch, the most important entity of differential diagnosis, with primarily trisomy 21 and 22q11 microdeletion.

The patient delivered the baby by $\mathrm{C}$-section at 37 weeks of gestation in a second-degree maternity and the neonate deceased two days after delivery. Interestingly, the other child born in the same family has a disorder belonging to the autism spectrum, and the father is diagnosed with hyperinsulinism syndrome.

The parents had not allowed the pathology examination, but double aortic arch being an isolate anomaly found during the ultrasound prenatal examination, we could suppose that this was the cause of sudden neonatal death. The

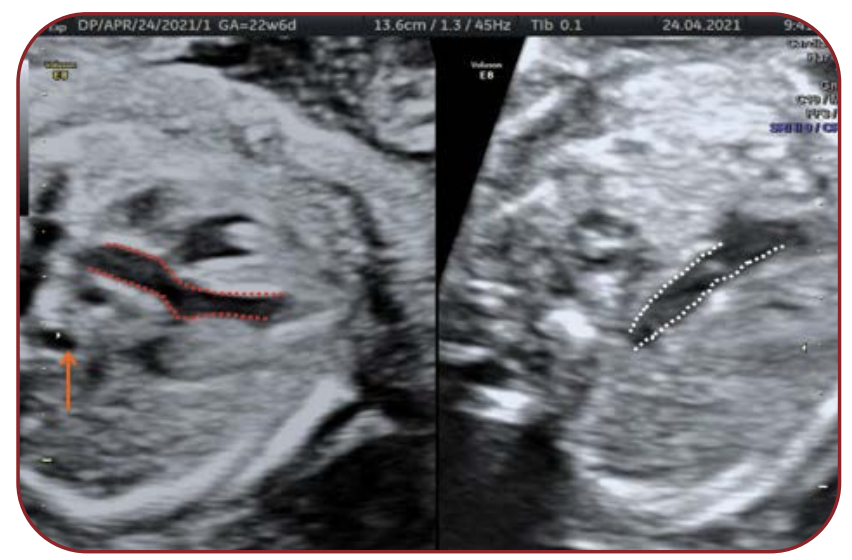

FIGURE 1. Double aortic arch case. Normal great vessel emergences, 2D standard images, sustain the bifurcation of the aortic arch anteriorly to the trachea, from the ascending aorta. The descending aorta (orange arrow); left ventricular outflow tract (red); right ventricular outflow tract (white)

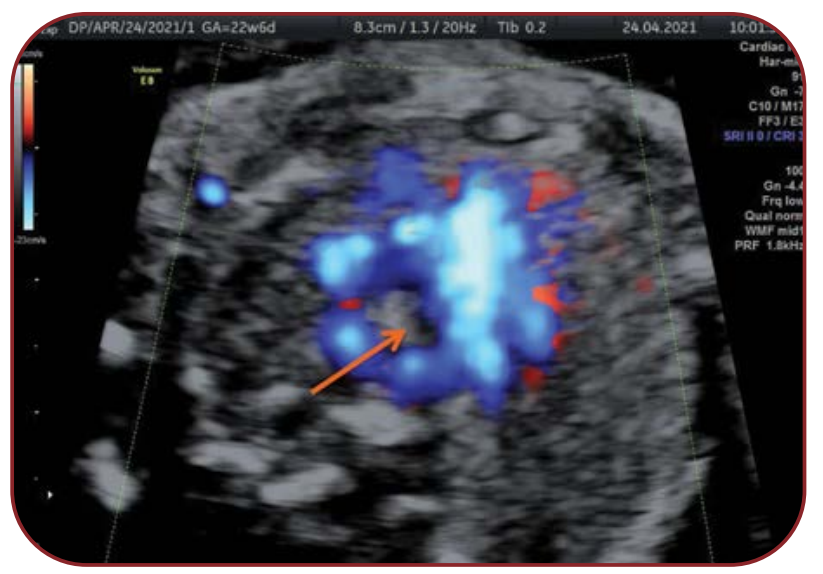

FIGURE 2. Double aortic arch case. Color Doppler vascular ring in transverse view of the fetal thorax at the level of the three-vessel and trachea view. The arrow indicates the trachea

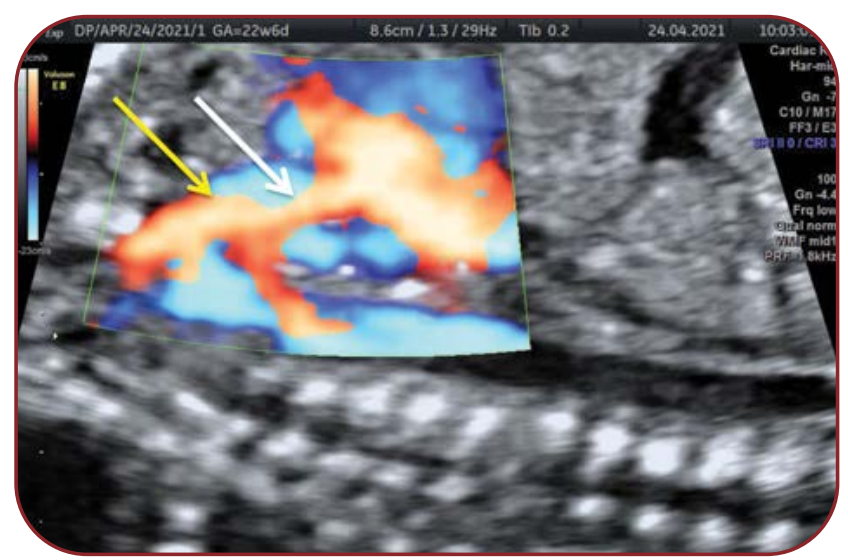

FI GURE 3. Double aortic arch case. Color Doppler longitudinal section of double aortic arch; the right arch is larger (yellow arrow) than the left hypoplastic one (white arrow) and is located more cephalic 


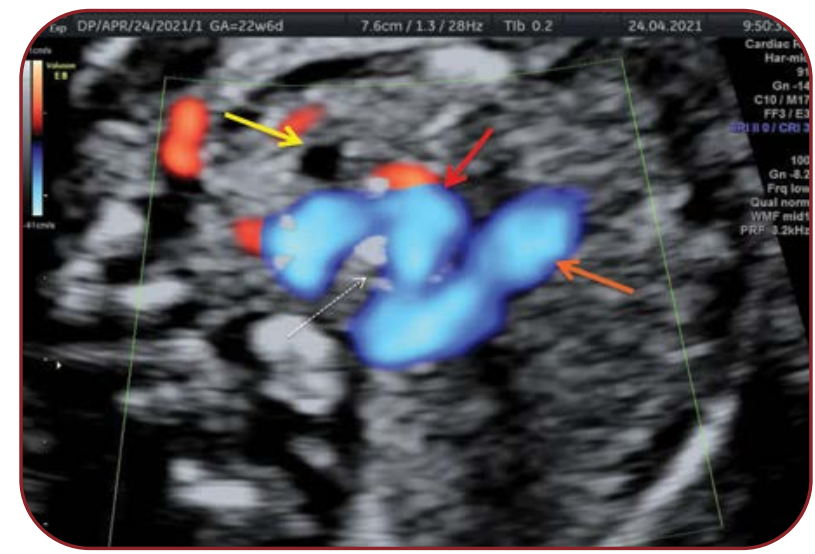

FIGURE 4. Double aortic arch case. Color Doppler in transverse plan of the fetal thorax at the level of ductus arteriosus. The bifurcation of the aorta into a right and left aortic arch (red arrow); superior vena cava (yellow arrow); trachea (white arrow); pulmonary artery (orange arrow)

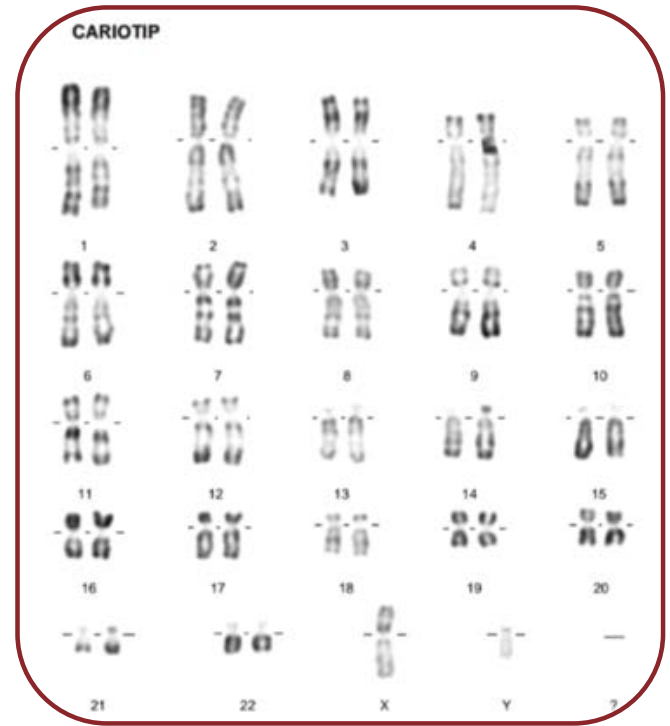

FIGURE 5. Normal karyotype of double aortic arch case

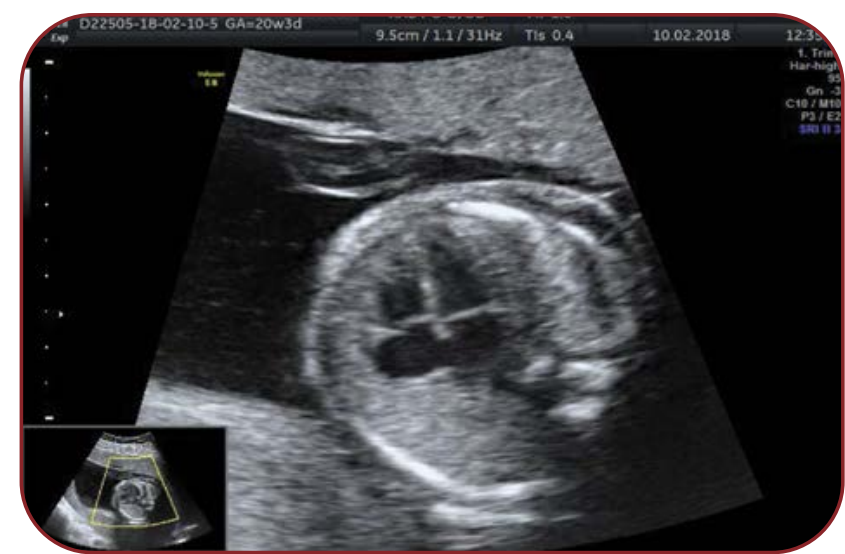

FIGURE 6. Right aortic arch associating an aberrant left subclavian artery and a left-sided ductus arteriosus case. Normal two-dimensional four-chamber view

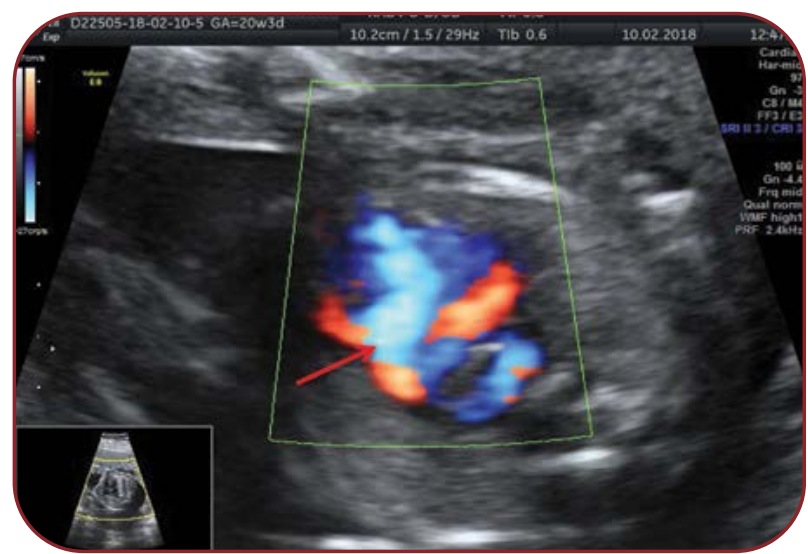

FIGURE 7. Right aortic arch associating an aberrant left subclavian artery and a left-sided ductus arteriosus case. The ascending aorta takes an opposite direction from the usual normal one (red arrow)

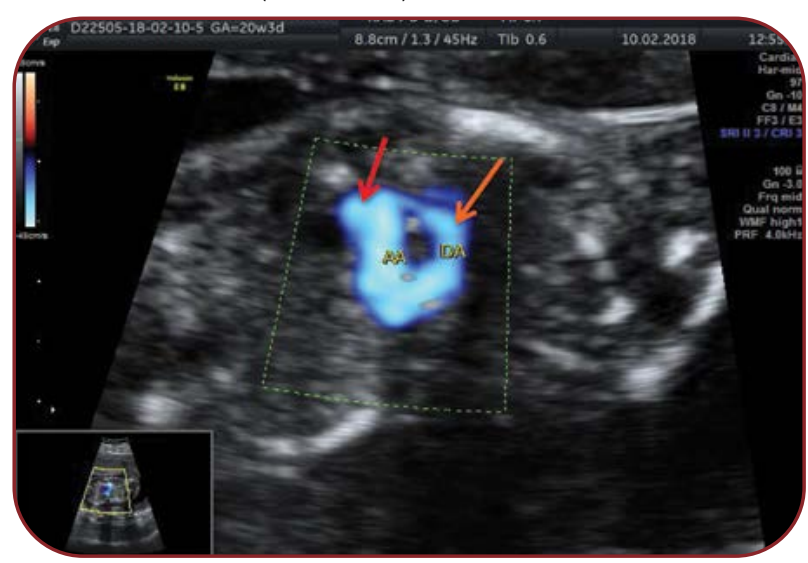

FIGURE 8. Right aortic arch associating an aberrant left subclavian artery and a left-sided ductus arteriosus case. Color Doppler vascular ring in transverse view of the fetal thorax at the level of the "V"- sign view. The image is completely different from the one presented in the case of double aortic arch. Aorta (red arrow) and pulmonary artery (orange arrow)

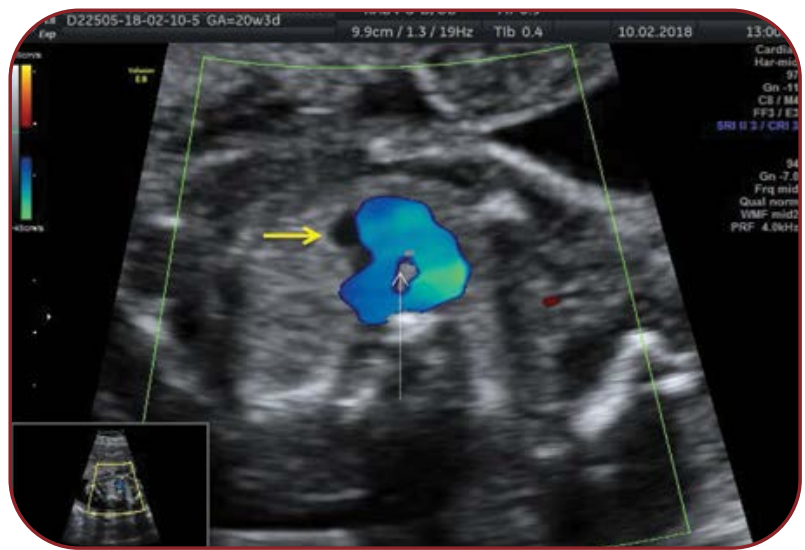

FIGURE 9. Right aortic arch associating an aberrant left subclavian artery and a left-sided ductus arteriosus case. HD flow vascular ring. Ductus arteriosus arises at the origin of the aberrant subclavian artery and takes an anterior course for connecting to the pulmonary artery. Superior vena cava (yellow arrow); trachea (white arrow) 
natural evolution of double aortic arch implies significant morbidity and in obscure cases, sudden death can occur from airway compromise even from the first days of life when ventilation support is not available.

\section{DISCUSSIONS}

$\mathrm{T}$ here is a broad spectrum of congenital variants and malformations of the aortic arch and it is mandatory to recognize them in order to improve the prognosis and management. Prenatal imaging is one of the most useful tools for an accurate diagnosis and ultrasound is the most used diagnostic procedure in obstetrics, with many well-known advantages (11).

Although fetal echography is typically used for diagnosis, Salehi D et al mention in their study that fetal cardio-MR is a useful complementary tool to ultrasonography in referred cases and it can influence the delivery planning and postnatal care (7). In our case, ultrasound was sufficient for diagnosis, without the need for other imagistic methods.

For an accurate diagnosis it is compulsory to know and understand the embryology and imaging spectrum of aortic arch variants. Hanneman et al state that the differentiation of an incomplete double aortic arch with distal left arch atresia from a right aortic arch with mirror image branching can be challenging and knowledge of the characteristic imaging findings of incomplete double aortic arch with distal left arch atresia is important, as this entity forms a complete vascular ring (unlike a right aortic arch with mirror image branching) (5).

Almost $90 \%$ of vascular ring cases are due to a double aortic arch or a right aortic arch associating an aberrant left subclavian artery and a left-sided ductus arteriosus (10, 12-14). For a deep and entire differentiation, we also present four images - from our personal collection - of the second cause of vascular ring formation by standard ultrasound examination sections during second trimester screening (Figures 1-4 versus Figures 6-9).

As we stated above, $22 q 11$ deletion is the most common chromosomal abnormality associated with double aortic arch. Although the karyotype was normal in our case, the literature reports many cases that prove a significant association between deletions of chromosome 22q11 and aortic anomalies, as showed by McElhinney DB et al in their study (15).

Guo Qiao YK et al mentioned that identifying other intracardiac and extracardiac congenital malformations or chromosomal anomalies was essential for prognostic evaluation and prenatal counseling (2).

The highly associated rate of genetic anomalies with aortic arch pathology described in the literature and the risk for respiratory arrest due to dynamic compression on the trachea may explain the risk for death among these patients.

\section{CONCLUSIONS}

ouble aortic arch represents a variant from the broad spectrum of congenital aortic arch anomalies, and knowledge of embryology and imaging spectrum of aortic arch anomalies is essential for an accurate antenatal diagnosis and therefore, a correct clinical management. The lambda-sign described for the double aortic arch and $U$-sign of right aortic arch can be seen from the first trimester ultrasound scan, but regardless the diagnosis timing, the key of good outcome of these cases is the prenatal ultrasound diagnosis that offers time for not only scheduling cardiac surgery of severe cases but also - and most of all - targeting the birth planning into a third degree maternity capable to manage the respiratory neonate emergencies.

Conflicts of interest: none declared.

Financial support: none declared.

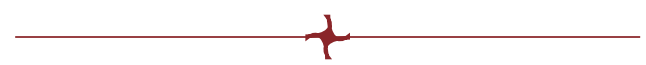

\section{R}

1. Satyapal KS, Lazarus L, Shama D. Double aortic arch: an unusual congenital variation.

Surgical and Radiologic Anatomy
2013;35:125-129.

2. Guo Qiao, Yifan Kong, Shi Zeng, et al. Fetal double aortic arch: prenatal sonographic and postnatal computed tomography angiography features, associated abnormalities and clinical outcomes.

BMC Pregnancy and Childbirth 2020;20:1-10. 
3. Hunter LN, Callaghan K, Patel L, et al. Prenatal echocardiographic diagnosis of double aortic arch.

Ultrasound in Obstetrics \& Gynecology 2015;45:483-485.

4. Ramos-Duran L, Nance JW Jr, Schoepf UJ, et al. Developmental aortic arch anomalies in infants and children assessed with CT angiography. American Journal of Roentgenology 2012;198:W466-W474.

5. Hanneman Kate, Newman Beverly, Chan F. Congenital variants and anomalies of the aortic arch. Radiographics 2017;37:32-51.

6. Trobo D, Bravo C, Alvarez Teresa, et al. Prenatal sonographic features of a double aortic arch: literature review and perinatal management. Journal of Ultrasound in Medicine 2015;34:1921-1927.

7. Salehi D, Fricke Katrin, Bhat M, et al.
Utility of fetal cardiovascular magnetic resonance for prenatal diagnosis of complex congenital heart defects. JAMA Network Open 2021;4:e213538-e213538.

8. Yerlikaya G, Efetürk T, Springer Stephanie, Reischer Theresa. Prenatal detection of right aortic arch." Archives of gynecology and obstetrics 2019;299:933-938.

9. Woods RK, Sharp RJ, Holcomb GW $3^{\text {rd }}$, et al. Vascular anomalies and tracheoesophageal compression: a single institution's 25-year experience. Ann Thorac Surg 2001;72:434.

10. Humphrey C, Duncan K, Fletcher S. Decade of experience with vascular rings at a single institution. Pediatrics 2006;117:e903.

11. Abramowicz JS. Benefits and risks of ultrasound in pregnancy. In: Seminars in perinatology, Vol. 37,
WB Saunders, 2013, pp 295-300.

12. Shah RK, Mora BN, Bacha E, et al. The presentation and management of vascular rings: an otolaryngology perspective.

INT J Pediatr Otorhinolaryngol 2007;71:57.

13. Woods RK, Sharp RJ, Holcomb GW $3^{\text {rd }}$, et al. Vascular anomalies and tracheoesophageal compression: a single institution's 25-year experience. Ann Thorac Surg 2001;72:434.

14. Backer CL, Mavroudis C, Rigsby CK, Holinger LD. Trends in vascular ring surgery.

J Thorac Cardiovasc Surg 2005;129:1339.

15. McElhinney DB, Clark BJ, Weinberg PM, et al. Association of chromosome 22q11 deletion with isolated anomalies of aortic arch laterality and branching.

Journal of the American College of Cardiology 2001;37:2114-2119. 